\title{
Introducing Nano-FTIR - Imaging and Spectroscopy at 10nm Spatial Resolution
}

\author{
Tobias Gokus ${ }^{1}$, Florian Huth ${ }^{1}$ and Andreas Huber ${ }^{1}$ \\ ${ }^{1 .}$ Neaspec GmbH, Bunsenstrasse 5, 82152 Planegg (Munich), Germany
}

Scattering-type scanning near-field optical microscopy systems (s-SNOM) allows to overcome the diffraction limit of light enabling optical measurements, imaging as well as spectroscopy, at a spatial resolution of $10 \mathrm{~nm}$ not only at visible frequencies but also in the infrared or terahertz spectral range.

S-SNOM employs an externally-illuminated sharp metallic AFM tip to create a nanoscale hot-spot at its apex [1]. The optical tip-sample near-field interaction is determined by the local dielectric properties (complex refractive index) of the sample and detection of the elastically tip-scattered light yields nanoscale resolved near-field images simultaneous to topography.

Here we demonstrate that, based on the sSNOM concept, it is possible to perform molecular vibrational spectroscopy (nano-FTIR) of complex organic and inorganic nanostructures with 10-20nm spatial resolution can be realized [2]. For nano-FTIR spectroscopy the AFM tip of the sSNOM system is illuminated by a mid-IR broadband laser source while the light which is elastically scattered back from the AFM tip is analyzed with the help of an asymmetric Michelson interferometer. Nano-FTIR spectroscopy yields simultaneously amplitude and phase spectra of the materials under study and ultimately allows for directly determining the corresponding absorption as well as reflection spectra (see Figure 1). Furthermore, we find that nano-FTIR absorption spectra correspond well with transmission far-field FTIR spectra and therefore can be directly used for chemical characterization of unknown materials. Other applications show characterization of embedded structural phases in biominerals [3] or organic semiconductors [4]. The universal nano-FTIR approach presented here also allows for tailored system configurations where the ultimate spectral coverage can be achieved by using synchrotron-based broadband IR light sources [5] or pump-probe measurements at 10nm spatial resolution [6].

Equipping s-SNOM systems with cw light sources we demonstrate that Mid-IR s-SNOM near-field imaging can be performed at time scales of 30-300s per image. Use of material-selective frequencies in the mid-IR spectral range can be exploited to fully characterize polymer blends [7] or phase change materials with nanometer-scale domains. Quantification of free-carrier concentration and carrier mobility in doped semiconductor nanowires [8] or visualization and analysis of localized and propagating plasmons across graphene nanostructures [9] is achieved by amplitude- and phase-resolved infrared near-field imaging. Especially, the latter application holds great promises for graphene quality analysis as is possible to highlight grain boundaries and electronic defects which are not observable in standard AFM topography images. We envision that correlative IR graphene plasmon s-SNOM imaging and near-field photocurrent imaging will be a powerful tool for studying the electronic properties of graphene devices.

Extending the concept of broadband-sSNOM spectroscopy to the THz-spectral range, we demonstrate nanoscale material characterization at THz-frequencies by coupling the free space beam of a THz-TDS system to a s-SNOM microscope [10,11]. Material specific near-field spectra of metals and highly doped semiconductors in the spectral range between $0.5-2.5 \mathrm{THz}$ as well as near-field images (at fixed delay) can be obtained. The presented results open the door to study nanoscale light-matter interaction in the THzspectral range for all materials systems that can be investigate by conventional AFM measurements. 
Further, due the reflective optics design typically employed in s-SNOM systems we envision that also timeresolved near-field pump-probe experiments in the $\mathrm{THz}$ spectral range can be realized in order to study e.g. the charge carrier dynamics in semiconductors but also in biological complexes with nanoscale resolution.

References:

[1] F. Keilmann, R. Hillenbrand, Phil. Trans. R. Soc. Lond. A 362, 787 (2004)

[2] F. Huth et al, Nano Lett. 12, 3973 (2012)

[3] S. Amarie, et al, Beilstein J. of Nanotech. 3, 312 (2012)

[4] C. Westermeier et al, Nature Comm. 5, 4101 (2014)

[5] P. Herrmann et al, Optics Expr. 21, 2913 (2013)

[6] M. Eisele et al, Nature Phot. 8, 841 (2014)

[7] T. Taubner et al, APL 85, 5064 (2004)

[8] J. Stiegler et a., Nature Comm. 3, 1131 (2012)

[9] J. Chen et al, Nature 487, 77 (2012)

[10] K. Moon et al, APL, 101, 011109 (2012)

[11] A. Huber et. al, Nano Lett., 8, 3766 (2008)

(a)
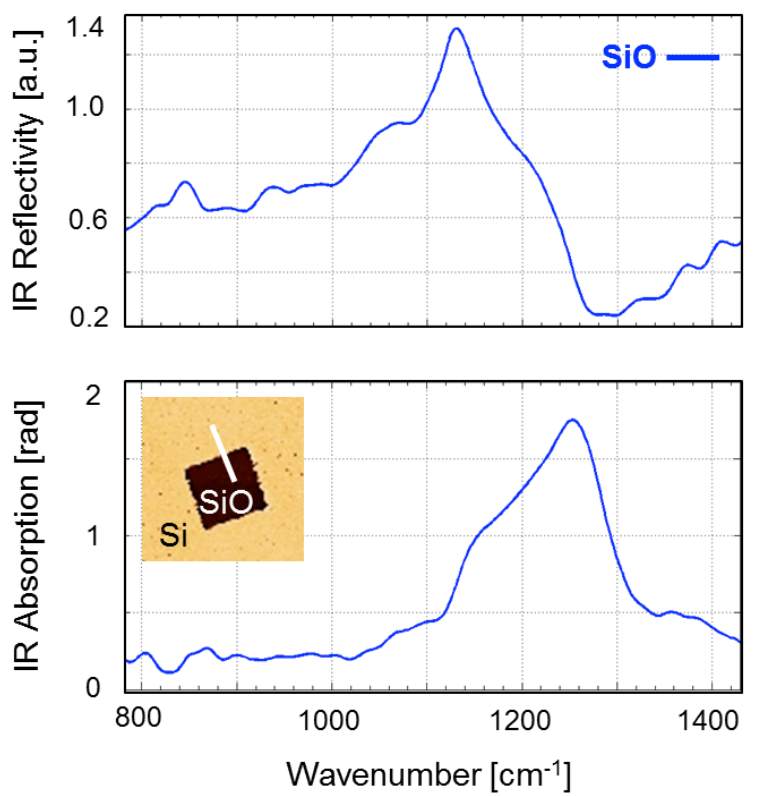

(b)
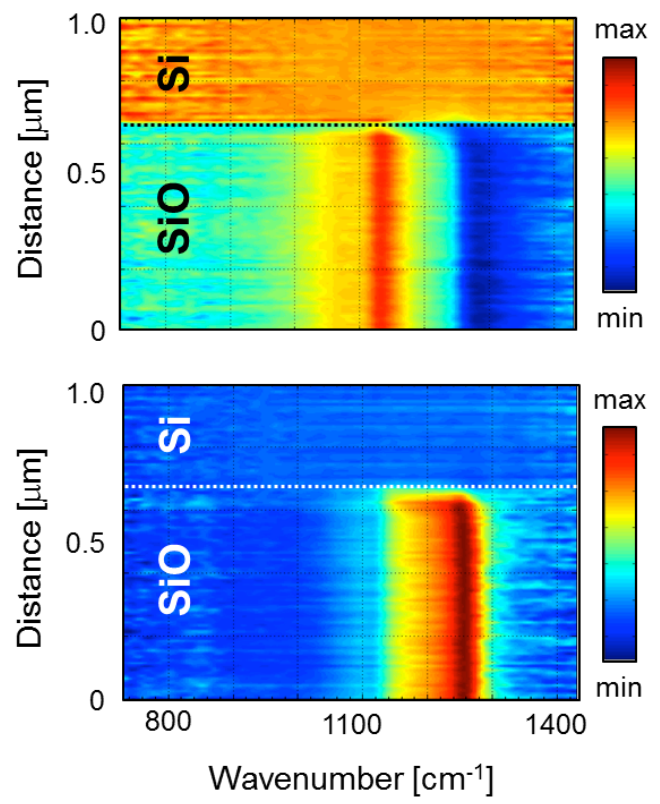

Figure 1. Nano-FTIR spectrum of a Si-SiO nano-structure. (a) Near-field reflectivity and absorption spectrum of SiO revealing a pronounced material-specific spectral signature. Detailed lineshape analysis allows identifying up to 5 distinct resonances at ca. 840, 1060, 1120,1210, and $1365 \mathrm{~cm}^{-1}$ determining the spectral signature. Inset: IR near-field image of $\mathrm{Si}-\mathrm{SiO}$ nanostructure at $1600 \mathrm{~cm}^{-1}$ where the $\mathrm{SiO}$ spectral signature was measured, exhibiting a pronounced contrast due to different refractive index of materials. White line marks position of line profile. (b) Near-field spectroscopic line profile across $\mathrm{SiO}$ and Si interface (raw data, i.e. no filtering applied). Spectroscopic measurement along $1 \mu \mathrm{m}$ distance (10 $\mathrm{nm}$ pixel size, 100 spectra, 30s/spectra) exhibits abrupt change of measured spectral signature of $\mathrm{SiO}$ to a homogeneous spectral response on $\mathrm{Si}$ at 20-30 nm spatial resolution. 